\title{
Swiss Learning Health System
}

\section{Stefan Boes ${ }^{a}$, Mirjam Brach ${ }^{a, b}$, Cornel Kaufmann ${ }^{a}$, Sarah Mantwilla, Sara Rubinellia,b, Gerold Stuckia,b, für das Konsortium der Projektpartner im SLHS}

a Universität Luzern, Seminar für Gesundheitswissenschaften und Gesundheitspolitik

b Schweizer Paraplegiker Forschung, Nottwil

Das Schweizer Gesundheitssystem zählt zu den besten weltweit. Um auch künftig den Herausforderungen im Gesundheitswesen effektiv und effizient begegnen zu können, bedarf es einer Infrastruktur und einer Kultur, in welcher die beste Evidenz systematisch verfügbar gemacht und genutzt wird und sich das System basierend auf einem ständigen Austausch zwischen Forschung, Politik und Praxis weiterentwickelt. Das Swiss Learning Health System soll dies in enger Zusammenarbeit mit den verschiedenen Akteuren im Gesundheitswesen institutionalisieren. Dieser Artikel stellt die Ziele und Mechanismen des Swiss Learning Health Systems dar vor dem Hintergrund nationaler und internationaler Initiativen zur Stärkung der Gesundheitsversorgung und zur Verbesserung der Gesundheit der Bevölkerung durch Lernzyklen.

\section{Hintergrund}

Moderne Gesundheitssysteme, wie das der Schweiz, werden in den nächsten Jahren mit zahlreichen Herausforderungen konfrontiert. Die Alterung der Bevölkerung, neue und veränderte Krankheitsbilder, der technische Fortschritt und wechselnde Anforderungen an die Gesundheitsberufe sind nur einige Beispiele dafür. Um den kommenden Herausforderungen effektiv und effizient zu begegnen, hat der Bundesrat die Strategie Gesundheit 2020 verabschiedet [1]. Diese legt

\section{Résumé}

Le système de santé suisse est l'un des meilleurs au monde. Pour que les défis sanitaires puissent continuer d'être relevés de manière efficace et performante, il faut une infrastructure et une culture dans lesquelles la meilleure donnée factuelle est systématiquement rendue accessible et exploitée et dans lesquelles le système se perfectionne sur la base d'un échange constant entre la recherche, la politique et la pratique. Le Swiss Learning Health System vise à institutionnaliser cette démarche en étroite collaboration avec les différents acteurs du système de santé. Cet article présente les objectifs et mécanismes du Swiss Learning Health System, dans un contexte d'initiatives nationales et internationales de renforcement des soins et d'amélioration de la santé de la population par des cycles d'apprentissage.

\section{Konsortium der Projektpartner}

und lokale Koordinatoren

- Universität Basel, Swiss Tropical and Public Health Institute (SwissTPH): Kaspar Wyss

- Université de Neuchâtel: Paul Cotofrei, Kilian Stoffel

- Università della Svizzera italiana (USI): Stefano Calciolari, Marco Meneguzzo

- Universität Luzern (Hauptantragsteller): Gerold Stucki, Stefan Boes, Adrian Loretan, Bernhard Rütsche

- Universität Zürich: Thomas Rosemann, Stefan NeunerJehle

- Scuola universitaria professionale della Svizzera italiana (SUPSI): Luca Crivelli, Carlo Di Pietro

Zürcher Hochschule für Angewandte Wissenschaften (ZHAW): Urs Brügger, Karin Niedermann, Astrid Schämann, Simon Wieser

Swiss School of Public Health (SSPH+): Nino Künzli

vier übergeordnete Handlungsfelder für gesundheitspolitische Massnahmen und Aktivitäten fest: 1. Lebensqualität, 2. Versorgungsqualität, 3. Chancengleichheit und 4. Transparenz. Ziel ist es, ein Gesundheitssystem zu entwickeln, das auf die spezifischen Bedürfnisse der Menschen ausgerichtet ist, zur Verbesserung der Gesundheit und des Wohlbefindens der Bevölkerung. Das Bundesamt für Gesundheit (BAG) unterstrich bereits in seinem Forschungskonzept Gesundheit 2013-2016 entsprechend die Notwendigkeit zur Stärkung der Ver- 
sorgungsforschung [2]. Hier hat die Schweiz noch immer Nachholbedarf. Laut BAG fehlt «die Koordination der Aktivitäten ebenso wie die Vernetzung der Akteure und eine umfassende Forschungsförderung» [2, S.24]. Trotz wichtiger Fortschritte, z.B. durch das Förderprogramm der Bangerter-Stiftung [3], erachtet das BAG auch im aktuellen Konzept 2017-2020 die weitere Entwicklung der Versorgungsforschung als zentral [4]. Die Schweizerische Akademie der Medizinischen Wissenschaften (SAMW) sieht die Versorgungsforschung neben der biomedizinischen und klinischen Forschung als dritten Ansatz der Gesundheitswissenschaften [5]. In ihrem Konzept zur Stärkung der Versorgungsforschung gibt die SAMW Empfehlungen "für den mittelbis langfristigen Ausbau von Forschungskompetenzen, Infrastrukturen, Datenlage sowie Nachwuchsförderung und Finanzierungsmöglichkeiten im Bereich Versorgungsforschung» [5, S. 8]. Neben der Notwendigkeit der Forschungsförderung im Rahmen eines nationalen Forschungsprogramms des Schweizerischen Nationalfonds (umgesetzt im NFP74 Gesundheitsversorgung, www.nfp74.ch) sieht die SAMW auch Bedarf für eine nationale Infrastruktur für Versorgungsforschung.

Das Projekt Swiss Learning Health System (SLHS) knüpft an diese Idee an. Unterstützt durch das Staatssekretariat für Bildung, Forschung und Innovation soll das SLHS eine neue Infrastruktur durch die Errichtung

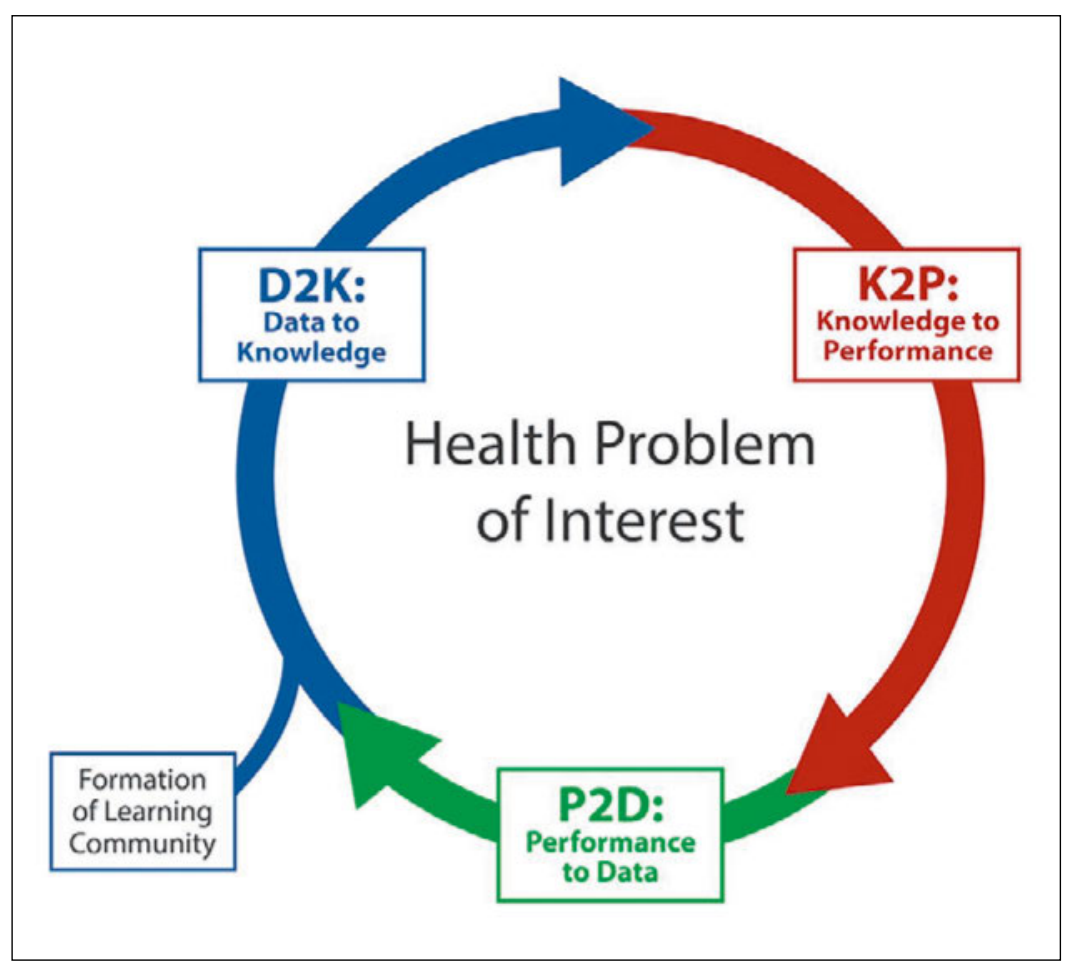

Abbildung 1: Aus Friedman et al. (2017) [9]. einer nationalen Plattform für Gesundheitssystem- und Versorgungsforschung schaffen. Das SLHS setzt sich zum Ziel, Politik und Praxis zu stärken sowie eine Kultur zu schaffen, in welcher wissenschaftliche Grundlagen kontinuierlich in das Gesundheitssystem integriert und wissenschaftsbasierte Lösungsansätze zu gesundheitspolitischen Fragestellungen erarbeitet und umgesetzt werden.

\section{Lernzyklen im Gesundheitswesen}

Die Grundlagen für die Entwicklung des SLHS bilden das Health Systems Framework der Weltgesundheitsorganisation (WHO) sowie das Konzept des Learning Health Systems (LHS) des Institute of Medicine bzw. der National Academy of Medicine in den USA. Die Idee eines LHS basiert auf der Annahme, dass ein Gesundheitssystem dann lernen kann, wenn es auf zyklische Prozesse zurückgreift. In diesen Prozessen werden für das Gesundheitssystem relevante Daten erfasst, welche als Grundlage dienen, um neue Evidenz zu generieren. Diese Evidenz wird zur Umsetzung in die Praxis transferiert und aus der Praxis generierte Daten sowie Erfahrungen aus der Umsetzung werden wiederum in den Zyklus eingespeist. Das Ziel eines solchen Zyklus ist es, effektive und effiziente Massnahmen zur Adressierung von spezifischen Problemen zu identifizieren um die Gesundheit einzelner Personen und der Bevölkerung im Ganzen zu verbessern [6-8]. Schematisch lässt sich dies wie in Abbildung 1 darstellen.

Während LHS-Strukturen seit 2007 vor allem in den USA aufgebaut wurden (z.B. CancerLinQ, PCORnet, Sentinel Initiative, oder das Health Care Systems Research Network), hat sich das Interesse an lernenden Gesundheitssystemen mittlerweile global ausgebreitet. Beispiele sind das TRANSFoRm Projekt unterstützt durch die Europäische Union, das European Institute for Innovation through Health Data (i HD), das Learning Healthcare Project in Grossbritannien, oder das Consortium for Asia Pacific Learning Health Systems (siehe auch [9]).

Damit ein Gesundheitssystem gemäss dem in Abb. 1 gezeigten Zyklus lernen kann, benötigt es einen konstanten Austausch zwischen Wissenschaft, Politik und Praxis. Dies ist einerseits eine Frage der Kommunikation und Dissemination von Forschungsergebnissen. Andererseits braucht es die Bereitschaft zu einem lösungsorientierten Diskurs aller Stakeholder, um Probleme zu identifizieren, zu verstehen und kollaborativ geeignete Lösungsvorschläge und Implementierungsstrategien zu erarbeiten. Der konstante und systematische Einbezug der verschiedenen Stakeholder im Gesundheitswesen ist essentielle Basis für ein lernendes 


\section{SLHS Cycle}

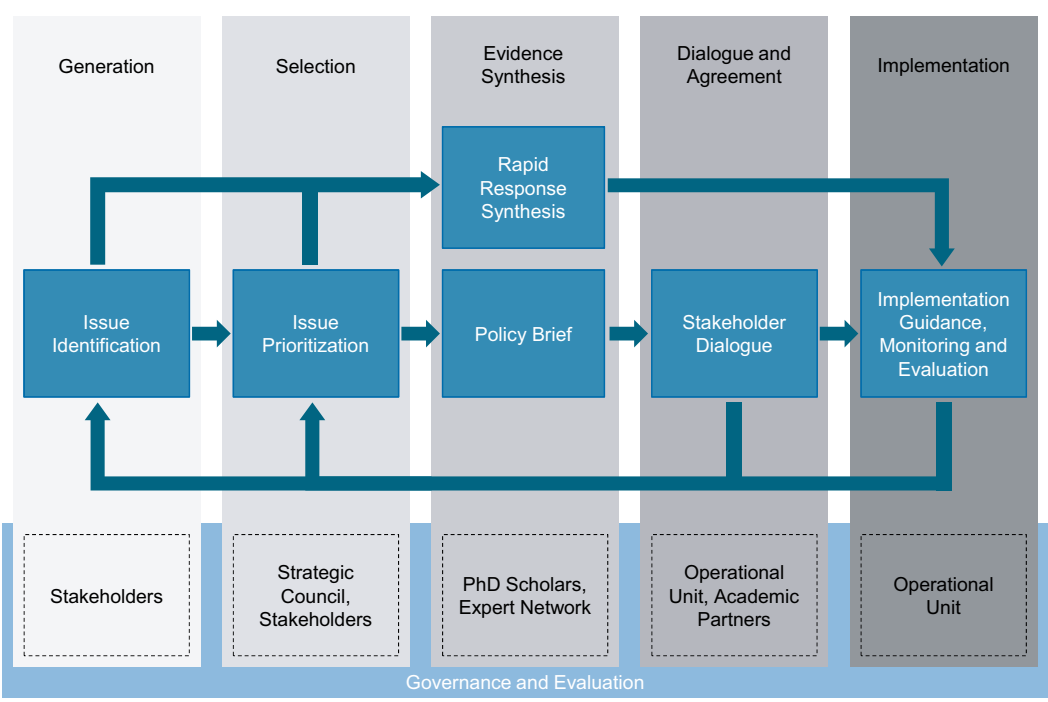

Abbildung 2: Brückenmechanismus im SLHS, eigene Darstellung

System. In einem lernenden System sind alle Akteure aufgerufen, sich mit Themen aktiv zu beteiligen. Die erfolgreiche Umsetzung eines LHS benötigt deshalb entsprechende Infrastrukturen und eine Kultur im Gesundheitssystem, in welcher Lernprozesse routinemässig angewandt werden. Vor diesem Hintergrund ist das SLHS in der Schweiz die erste Initiative weltweit, die ein lernendes Gesundheitssystem auf nationaler Ebene aufbaut.

\section{Ziele des Swiss Learning Health System}

Das SLHS verfolgt drei Hauptziele:

- Etablierung eines Brückenmechanismus zwischen Forschung, Politik und Praxis

- Förderung des Aufbaus wissenschaftlicher Kapazitäten für Gesundheitssystem- und Versorgungsforschung

- Management von standardisierten Gesundheitsinformationen

Konkret soll dies durch Anwendung von spezifischen Mechanismen erreicht werden.

\section{Mechanismen}

Die Etablierung eines Brückenmechanismus zwischen Forschung, Politik und Praxis steht im Zentrum des SLHS. Dieser Mechanismus kann durch folgenden Zyklus dargestellt werden (Abbildung 2):

\section{Problemidentifikation und Priorisierung}

Um aktuelle Herausforderungen im Gesundheitssystem zu identifizieren, muss ein systematischer und fortlaufender Dialog zwischen Forschung, Politik und Praxis stattfinden. Dazu soll einerseits der Austausch an bestehenden Tagungen und Konferenzen genutzt werden, andererseits sollen neue Formate integriert werden, wie z.B. Stakeholder Meetings oder Fokusgruppen. Dem Strategic Council des SLHS, in welchem aktuell alle bestehenden Partnerinstitutionen (siehe S.1) vertreten sind, kommt eine zentrale Rolle bei der Themenpriorisierung zu. Unter Berücksichtigung ethischer und rechtlicher Grundsätze sowie der Expertise der beteiligten Forschergruppen und eines breiten Netzwerkes aus der Praxis sollen Themen, die für das Schweizer Gesundheitssystem von Relevanz sind, frühzeitig erkannt und priorisiert werden.

\section{Evidenzsynthese}

Für priorisierte Themen werden sogenannte Evidenzsynthesen durch das SLHS erarbeitet. An diesem Prozess sind alle akademischen Partnerinstitutionen mit ihren Netzwerken beteiligt. Der Prozess umfasst die Entwicklung von Policy Briefs (als Input für Stakeholder Dialoge) oder Rapid Response Synthesen. Policy Briefs haben das Ziel, ein Thema bzw. ein bestehendes Problem mit allen dazugehörigen Kontextfaktoren $\mathrm{zu}$ beschreiben und zu erläutern. Gleichzeitig werden evidenzbasierte Lösungen für das Problem aufgezeigt und deren Kosten und Nutzen sowie mögliche Barrieren in der Umsetzung diskutiert. Bei spontan auftretenden Problemen und zeitnah erforderlichen Reaktionen stellt das SLHS alternativ Rapid Response Synthesen bereit. Gemäss dem aktuellen Erkenntnisstand der Forschung sollen diese in kurzer Form die besten Lösungsansätze für ein bestimmtes Problem zusammenfassend darstellen.

\section{Stakeholder Dialog}

Entscheidungsfindung in der Gesundheitspolitik ist ein partizipativer Prozess, in welchem sich Stakeholder über geeignete Vorgehensweisen für bestimmte Themen einigen. Diese Interaktion findet jedoch nicht in einem Vakuum statt, sondern wird durch verschiedene Faktoren beeinflusst wie z.B. institutionelle Rahmenbedingungen, Interessensgruppen, Wertvorstellungen und Zeitrestriktionen, aber auch Probleme in der Kommunikation. Als Folge dessen scheitert häufig der Wissenstransfer und die Verarbeitung neuer Evidenz. Das SLHS zielt darauf ab, einen Mechanismus bereitzustellen, der eine evidenzbasierte Gesundheitspolitik unterstützt, den Stakeholder Dialog. Konzeptionell angelehnt an das McMaster Health Forum (https:// www.mcmasterhealthforum.org) stellt der Stakeholder Dialog einen strukturierten Kommunikationsprozess dar, in welchem Stakeholder a) sich über das zu disku- 
tierende Problem einig werden, b) ihre Positionen zu den verschiedenen Lösungsalternativen darlegen, und c) eine Einigung zur besten Vorgehensweise erzielen. Stakeholder Dialoge sind wirkungsvolle Mechanismen, für die Identifizierung und Priorisierung von evidenzbasierten Massnahmen, die erfolgreich im Gesundheitssystem umgesetzt werden können. Zusätzlich bieten Stakeholder Dialoge die Möglichkeit, neue Themen für eine Forschungsagenda zu identifizieren, welche die Bedürfnisse und dringenden Fragen im Gesundheitssystem anspricht.

\section{Unterstützung in der Implementierungsphase} Wesentlich für ein LHS ist die fortlaufende Evaluation von Änderungen. Das SLHS begleitet deshalb die Implementierung von Massnahmen, auf welche sich Stakeholder im Rahmen eines Dialogs geeinigt haben, oder welche basierend auf Rapid-Response-Synthesen entschieden wurden. Die gesammelten Daten werden analysiert, den Stakeholdern bereitgestellt und fliessen im Sinne eines lernenden Gesundheitssystems wieder in die Forschung ein.

\section{Aufbau wissenschaftlicher Kapazitäten}

Der Aufbau wissenschaftlicher Kapazität ist das zweite wichtige Ziel des SLHS. Finanziert durch Eigenmittel der akademischen Partnerinstitutionen und die projektgebunden Beiträge des Bundes werden anfänglich 20 Promotionsstipendien für 4 Jahre vergeben. Die Doktorierenden nehmen eine zentrale Rolle bei den SLHS-Aktivitäten ein. Sie tragen bei der Erarbeitung von Policy Briefs aktiv zum SLHS-Zyklus bei. Zusätzlich profitieren die Doktorienden von den qualitativ hochstehenden Kursangeboten der Swiss School of Public Health (SSPH+). Mit dem Ziel der Identitätsbildung absolvieren die Doktorierenden spezifische Kurse in SLHS-nahen Themen. Mit einem strukturierten Ausbildungsprogramm werden trotz unterschiedlicher akademischer Verankerungen Fähigkeiten vermittelt, welche die Grundlage für ein gemeinsames Verständnis eines lernendes Gesundheitssystem darstellen.

Daneben soll ein LHS Enhancement Program die bestehenden Ausbildungsmöglichkeiten in der Schweiz ergänzen mit spezifischen Angeboten zu den Prinzipien eines lernenden Gesundheitssystems. Dazu gehören Kurse zu SLHS-spezifischen Themen wie dem Stakeholder-Dialog oder Workshops und Tagungen, in welchen die SLHS-Aktivitäten präsentiert und diskutiert werden. Ziel ist die Entwicklung und Förderung einer gemeinsamen Sprache und die Schaffung einer Kultur für ein lernendes Gesundheitssystem.

\section{Management von standardisierten Gesundheitsinformationen}

Gemäss WHO ist die Verfügbarkeit von Gesundheitsinformationen ein zentraler Baustein von Gesundheitssystemen. Die Erhebung, Analyse und Verbreitung von verlässlichen und aktuellen Informationen ist daher eine wichtige Voraussetzung für den erfolgreichen Betrieb eines (lernenden) Gesundheitssystems auf allen Ebenen - Forschung, Politik und Praxis. In Zusammenarbeit mit der WHO, nationalen und internationalen Fachgesellschaften aus Medizin und Gesundheitsberufen setzt sich das SLHS deshalb zum Ziel, nationale Datenstandards zu erarbeiten, die a) eine umfassende Beschreibung des Gesundheitssystems und b) ein umfassendes Reporting von Gesundheit und Funktionsfähigkeit von Individuen und der Gesellschaft erlauben. Datenstandards können als Brücke zwischen verbreiteten klinischen und auf Eigenangaben basierenden Messinstrumenten dienen. Ein entscheidender Aspekt der Entwicklung von Datenstandards ist die Verbesserung der Datenqualität. Ein Health Data Warehouse soll existierende Datenquellen (Kohortenstudien, Befragungen, Spital- und Versicherungsdaten, Statistiken) identifizieren, beschreiben, für Analysen nutzbar und vergleichbar machen sowie vernetzen. Damit soll die Datennutzung für Forschende innerhalb und ausserhalb des SLHS standardisiert und erleichtert werden. In diesem Sinne unterstützt das SLHS das 2013 verabschiedete Manifest «Bessere Gesundheitsdaten für ein effizienteres $\mathrm{Ge}$ sundheitswesen» von Public Health Schweiz.

\section{Partnernetzwerk und «Getting Involved»}

Die folgenden Universitäten und Fachhochschulen sind mit Eigenmitteln für Promotionsstipendien an der Entwicklung des SLHS beteiligt:

- Universität Basel: Schweizerisches Tropen- und Public-Health-Institut (assoziiertes Institut)

- Université de Neuchâtel

- Università della Svizzera italiana

- Universität Luzern

- Universität Zürich

- Scuola universitaria professionale della Svizzera italiana

- Zürcher Hochschule für Angewandte Wissenschaften

Neben den bestehenden Partnernetzwerken und -institutionen wie z.B. der Swiss School of Public Health, dem Institut für Hausarztmedizin und Community Care 
in Luzern oder der Schweizer Paraplegiker-Forschung in Nottwil ist das SLHS bestrebt, ein breites Netzwerk im Sinne einer schweizweiten Plattform für Gesundheitssystem- und Versorgungsforschung, Politik und Praxis aufzubauen. Die Partnerinstitutionen des SLHS bringen ihre Themen in die Entwicklung des SLHS ein und tragen zu einer nachhaltigen Umsetzung der Idee eines lernenden Gesundheitssystems in der Schweiz bei.

\section{Bildnachweise}

Abbildung 1: @ Schattauer GmbH, Verlag für Medizin und Naturwissenschaften, Stuttgart

Abbildung 2: @ Stefan Boes, eigene Darstellung

\section{Referenzen}

1 Bundesamt für Gesundheit (2013): Gesundheit 2020. Die gesundheitspolitischen Prioritäten des Bundesrates. Bundesamt für Gesundheit (BAG), Bern. Januar 2013.

2 Bundesamt für Gesundheit (2012): Forschungskonzept Gesundheit 2013-2016. Bundeamt für Gesundheit (BAG), Bern. Januar 2012.

3 Röthlisberger M, Amstad H (2017): Förderprogramm «Versorgungs forschung im Gesundheitswesen». Schweizerische Ärztezeitung 98(3):59-61.

4 Bundesamt für Gesundheit (2015): Forschungskonzept Gesundheit 2017-2020. Bundeamt für Gesundheit (BAG), Bern. Dezember 2015.

5 Schweizerische Akademie der Medizinischen Wissenschaften (2014): Stärkung der Versorgungsforschung in der Schweiz Swiss Academies Reports 9(1).

6 Institute of Medicine (2007): The Learning Healthcare System: Workshop Summary. Washington, DC: The National Academies Press.

Korrespondenz:

Universität Luzern SLHS Operational Unit Prof. Dr. Stefan Boes Frohburgstrasse 3 CH-6002 Luzern Tel.: 0412295959 slhs[at]unilu.ch www.slhs.ch
7 Institute of Medicine (2013): Best Care at Lower Cost: The Path to Continuously Learning Health Care in America. Washington, D.C.: The National Academies Press.

8 National Academy of Medicine (NAM). Learning Health System Series. Siehe https://nam.edu/programs/value-science-drivenhealth-care/learning-health-system-series

9 Friedman CP, Rubin JC, Sullivan KJ (2017): Toward an Information Infrastructure for Global Health Improvement. IMIA Yearbook of Medical Informatics 2017.

\section{Vorschau}

Von Shared Decision Making bis zum ultimativen Entscheidunterstützungssystem

Der 14. Schweizerische Kongress für Gesundheitsökonomie und Gesundheitswissenschaften (SKGG) vom 3. November 2017 im Universitätsspital Bern befasst sich mit der Thematik und Problematik der Entscheidfindung im Gesundheitssystem. In fünf Keynotes und mehreren Parallelveranstaltungen wird versucht, dieThematik strukturiert einzugrenzen und möglichst konkret darzulegen. Dabei geht es nicht nur um die evidenzbasierte klinische Entscheidfindung, sondern auch um den Patienten und dessen Selbstbestimmung und Gesundheitskompetenz. Anderseits wird auf die Interprofessionalität und die Interdisziplinarität eingegangen, die ebenfalls zur Entscheidfindung einen wesentlichen Beitrag erbringen können, da davon ausgegangen werden muss, dass das monodisziplinäre Denken und Handeln den heutigen Anforderungen nicht mehr entspricht. Das Bindeglied zwischen Arzt und Patient bildet sodann das "Shared Decision Making", die gemeinsame Entscheidfindung zumindest von Arzt und Patient - generell aber von Anbietern und Nutzern von gesundheitsbezogenen Dienstleistungen. Zum Kongressabschluss wird auf das ultimative Entscheidunterstützungssystem, das "Learning Health System" eingegangen. Im Rahmen des Zukunftsforums Gesundheit, eines Parallelgefässes des Kongresses, wird das Swiss Learning Health System erläutert - dies nebst dem Referat «Künstliche Intelligenz und Roboter im Gesundheitsbereich: Hoffnung mit Grenzen?». Weitere Informationen unter: www.skgg.ch 